\title{
Muscle attachment to the condylar process of mandible: anatomical considerations
}

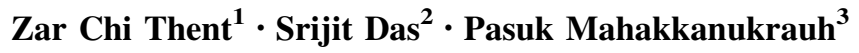

Received: 9 December 2015 / Accepted: 1 February 2016/Published online: 13 February 2016

(C) Springer-Verlag France 2016

We read with much interest the interesting article entitled 'An anatomic study of the attachments on the condylar process of the mandible: muscle bundles from the temporalis' by Sakaguchi-Kuma et al. [3]. At this juncture, we wish to share our scientific views on this published article.

The methods used by the authors are praiseworthy. No inclusion or exclusion criteria were mentioned in the study. It may be mentioned that presence of otological or neurological disease, or any history of past trauma or past surgical history may influence the muscle fibre alignment and it is mandatory to consider such. There are reports that condylar hypoplasia may also change the muscle tonus [1].

The average age of the cadavers was 79.3 years which means the subjects were very old. An important aspect to consider is the hardness of the food which was consumed by the deceased. With increase in age and hardness of food, the muscle architecture may change accordingly. A research study reported the fact that human jaw muscles have unique fibre type and undergo region-specific changes with increase in age [2]. All these facts should have been highlighted in detail.

It was interesting to note three references being cited in the results section which is otherwise not a common

Srijit Das

das_srijit23@rediffmail.com; drsrijit@gmail.com

1 Anatomy Unit, Faculty of Medicine, Universiti Teknologi Mara (UiTM), Sungai Buloh Campus, Jalan Hospital, 47000 Sungai Buloh, Selangor, Malaysia

2 Department of Anatomy, Universiti Kebangsaan Malaysia Medical Centre, Jalan Yaacob Latif, Bandar Tun Razak, 56000 Cheras, Kuala Lumpur, Malaysia

3 Forensic Osteology Research Centre, Faculty of Medicine, Chiang Mai University, Chiang Mai, Thailand phenomenon. A thorough discussion of the embryological reasons for the temporalis muscle fibre variations may bear much importance.

The authors describe that the muscle insertion may correspond to the shape of the bone which is again a debatable fact. In fact a strong mechanical pull by a muscle results in formation of any ridge or bony elevations in any bone. An example is the gluteus maximus muscle which produces the prominent gluteal tuberosity. Biomechanics of the muscle with regard to present findings should have been elaborated in detail.

Overall, it is an interesting article. We congratulate the authors for the meticulous study and thank the editor for publishing such an interesting study with clinical implications.

\section{Compliance with ethical standards}

Conflict of interest None to declare.

\section{References}

1. Arun T, Kayhan F, Kiziltan M (2002) Treatment of condylar hypoplasia with distraction osteogenesis: a case report. Angle Orthod 72:371-376

2. Monemi M, Kadi F, Liu JX, Thornell LE, Eriksson PO (1999) Adverse changes in fibre type and myosin heavy chain compositions of human jaw muscle vs. limb muscle during ageing. Acta Physiol Scand 167:339-345

3. Sakaguchi-Kuma T, Hayashi N, Fujishiro H, Yamaguchi K, Shimazaki K, Ono T, Akita K (2015) An anatomic study of the attachments on the condylar process of the mandible: muscle bundles from the temporalis. Surg Radiol Anat. doi:10.1007/ s00276-015-1587-4 